\title{
The impact of angiotensin receptor blockers on arterial stiffness: a meta-analysis
}

\author{
Feng Peng ${ }^{1,4}$, Hongming Pan ${ }^{2,4}$, Bin Wang ${ }^{2}$, Jinxiu Lin ${ }^{1}$ and Wenquan Niu ${ }^{3}$
}

Some studies reported a protective role of angiotensin receptor blockers (ARBs) against arterial stiffness. Therefore, we performed a meta-analysis of published clinical trials to systematically assess the impact of ARBs on arterial stiffness as measured by using pulse wave velocity (PWV). Eligible articles were identified by searching PubMed, EMBASE, Cochrane, Wanfang and CNKI databanks before 31 July 2014. The data were extracted independently and in duplicate. Forty articles including 53 clinical trials qualified, including 1650 and 1659 subjects in ARB treatment and control groups, respectively. Overall reductions in carotid-femoral PWV (cfPWV) and brachial-ankle PWV (baPWV) were statistically significant, with an average of $-42.52 \mathrm{~cm} \mathrm{~s}^{-1}$ (95\% Cl: -81.82 to $\left.-3.21 ; P=0.034\right)$ and $-107.08 \mathrm{~cm} \mathrm{~s}^{-1}$ (95\% Cl: -133.98 to $\left.-80.18 ; P<0.0005\right)$, respectively, after receiving ARBs. Subgroup analysis by ARB type revealed that telmisartan (weighted mean difference or WMD $\left.=-100.82 \mathrm{~cm} \mathrm{~s}^{-1} ; P<0.0005\right)$ and valsartan (WMD $\left.=-104.59 \mathrm{~cm} \mathrm{~s}^{-1} ; P<0.0005\right)$ significantly reduced baPWV, but only valsartan reduced cfPWV (WMD $=-65.58 ; P=0.030$ ). cfPWV was significantly reduced in comparisons of ARBs with placebo (WMD $=-79.65 \mathrm{~cm} \mathrm{~s}^{-1} ; P=0.001$ ), and baPWV was significantly reduced with calcium channel blockers (WMD $=-130.74 \mathrm{~cm} \mathrm{~s}^{-1} ; P<0.0005$ ). There were low probabilities of publication bias. Taken together, our findings support the important role of ARB treatment in improving arterial stiffness.

Hypertension Research (2015) 38, 613-620; doi:10.1038/hr.2015.51; published online 9 April 2015

Keywords: angiotensin receptor blocker; arterial stiffness; blood pressure; meta-analysis; pulse wave velocity

\section{INTRODUCTION}

It is well established that arterial stiffness is an independent predictor for future cardiovascular events and all-cause mortality in general populations. ${ }^{1-3}$ Mounting evidence suggests that the renin-angiotensin system (RAS) has a pivotal role in the progression of arterial stiffness, and blockade of this system can alleviate its progression. ${ }^{4,5}$ Angiotensin II receptor antagonists, which are known as angiotensin receptor blockers (ARBs), are a group of pharmaceuticals that inhibit the negative impact of angiotensin II. Angiotensin II is a potent vasoactive peptide on the endothelium in RAS, which lowers blood pressure and improves arterial elasticity. ${ }^{6,7}$ The beneficial impact of ARBs on arterial stiffness extends beyond the resulting blood pressure reduction. ${ }^{8}$ However, the exact mechanisms underlying the ARBdependent improvement in arterial stiffness remain poorly understood.

The heart is the primary site of damage of elevated arterial stiffness. Heart contraction produces a pulse or energy wave that travels through the circulation. Arterial stiffness is predominantly reflected by the traveling speed of this pulse wave, which is termed pulse wave velocity (PWV). PWV is established as a simple, well-validated and reproducible technique of applanation tonometry. ${ }^{9}$ PWV measurement is a general clinical tool that has yielded some of the strongest evidence of the prognostic significance of large artery stiffening. Several clinical trials explored the potential impact of ARBs on arterial stiffness in various populations. ${ }^{10-13}$ However, a comprehensive evaluation of this impact is lacking. Therefore, we systematically performed a meta-analysis of all published clinical trials that assessed the impact of ARBs on arterial stiffness as measured by PWV compared with other types of drugs.

\section{METHODS}

This meta-analysis conformed to the guidelines outlined in the Preferred Reporting Items for Systematic Reviews and Meta-analyses statement. ${ }^{14}$

\section{Search strategy}

We obtained potentially relevant articles by searching PubMed, EMBASE (Excerpta Medica Database), Cochrane (http://www.thecochranelibrary.com), Wanfang (http://www.wanfangdata.com.cn) and CNKI (China National Knowledge Infrastructure, http://www.cnki.net) as of 31 July 2014 by using

\footnotetext{
${ }^{1}$ Department of Cardiology, The First Affiliated Hospital of Fujian Medical University, Fuzhou, Fujian, China; ${ }^{2}$ Basic Medical Science College, Qiqihar Medical University, Qiqihar, Heilongjiang, China and ${ }^{3}$ State Key Laboratory of Medical Genomics, Ruijin Hospital, Shanghai Jiao Tong University School of Medicine, Shanghai, China

${ }^{4}$ These authors contributed equally to this work.

Correspondence: Professor J Lin, Department of Cardiology, The First Affiliated Hospital of Fujian Medical University, Chazhong Road 20, Fuzhou 350005, China.

E-mail: linjinxiu@medmail.com.cn

or Professor W Niu, State Key Laboratory of Medical Genomics, Ruijin Hospital, Shanghai Jiao Tong University School of Medicine, Ruijin Second Road 197, Shanghai 200025, China.

E-mail: niuwenquan_shcn@163.com

Received 23 October 2014; revised 4 February 2015; accepted 20 February 2015; published online 9 April 2015
} 
the subject terms 'pulse wave velocity,' 'arterial stiffness,' 'angiotensin receptor blocker,' 'angiotensin II antagonism' and 'sartan.' We additionally obtained the citations of relevant articles by reviewing the references of retrieved studies and review articles. We restricted search results to clinical trials on humans that were published in English or Chinese.

\section{Selection}

Two authors (Feng Peng and Wenquan Niu) independently read the titles and abstracts of all retrieved articles and assessed article eligibility. If an article could not be rejected with certainty from the title or abstract, we obtained its full text to decide whether data on the topic of interest were provided. If more than one publication was based on the same study group, the data from the most recent or complete article were extracted. If results were provided according to different drug types in either the treatment or control group, we treated the results separately.

\section{Inclusion/exclusion criteria}

Our analyses were restricted to clinical trials that met all of the following inclusion criteria: designed in a randomized manner and compared the ARB treatment with a placebo or other types of drugs on arterial stiffness as measured by PWV. Trials were excluded (one was sufficient for exclusion) if they were duplicate publications, conference abstracts, proceedings, case reports, case series, editorials, review articles or non-English and nonChinese articles.

\section{Data extraction}

Two authors (Feng Peng and Wenquan Niu) independently completed the data inclusion sheets using a standard Excel spreadsheet template (Microsoft, Redmond, WA, USA). We resolved disagreements during data extraction and reached consensus after a discussion and review of original articles.

We extracted detailed information for the impact of ARBs on arterial stiffness from each eligible article, including the first author, publication year, ethnicity and disease status of study patients, sample size in each arm, PWV type, study design, masking status, the type and dose of drugs used in each group, age, gender, body mass index, follow-up duration, levels and changes of systolic blood pressure (SBP) and diastolic blood pressure, pulse pressure, heart rate and PWV at the baseline and follow-up.

\section{Statistics}

Quantitative parameters were compared by using weighted mean difference (WMD) and 95\% confidence interval (95\% CI) when hinged on a randomeffects model by using the DerSimonian and Laird method ${ }^{15}$ for patients taking ARBs versus placebo or other drugs. Pearson correlation analysis was used to examine the relationship between quantitative parameters of interest. The inconsistency index $\left(I^{2}\right)$ statistic was calculated to assess between-study heterogeneity, and this statistic is defined as the percentage of the observed between-study variability that is because of heterogeneity rather than chance.

Predefined subgroup analyses were performed according to the types of drugs used in ARB treatment (mainly including valsartan, telmisartan, losartan, candesartan and irbesartan) and control (mainly including calcium channel blockers, angiotensin converting enzyme inhibitor or ACEI, placebo, non-RAS drugs, diuretics and $\beta$-blocker) groups to explore the potential sources of heterogeneity. Only subgroups involving two or more clinical trials were summarized in this meta-analysis.

We performed influential analysis by sequentially omitting each trial one at a time and computing differential estimates for the remaining trials to quantify the contribution of individual trials to the pooled estimates. We further resorted to meta-regression analysis to explore the other sources of heterogeneity from the divergence of baseline continuous characteristics across trials, including age, male percentage, body mass index and during of treatment.

We assessed the probability of publication bias by using Begg's test. The significance level was defined as $P<0.10$ for the $\chi^{2}$-test of $I^{2}$ and Begg's test. ${ }^{16}$ The statistical analyses described above were completed using STATA software (StataCorp, College Station, TX, USA, version 11.2 for Windows).

\section{RESULTS}

\section{Eligible trials}

We illustrated the characteristics of all eligible trials and study patients in Tables 1 and 2, respectively. A flow diagram schematizing the process of article exclusion for specific reasons is summarized in Figure 1. In total, 40 articles with 53 clinical trials qualified, including 1650 subjects in the ARB treatment group and 1659 subjects in the control. ${ }^{8,10-13,17-51}$ Of all clinical trials, 41 were performed in East Asians, and 12 trials were performed in Caucasians. For the ARB type, 24 trials adopted valsartan, 10 trials adopted telmisartan, 9 trials adopted losartan, 7 trials adopted candesartan and 3 trials adopted irbesartan. Control subjects included 20 trials that adopted calcium channel blockers, 9 trials that adopted ACEIs, 7 trials that adopted a placebo, 5 trials that adopted diuretics, 5 trials that adopted nonantihypertensive drugs, 3 trials that adopted beta blockers and 1 trial that adopted beraprost sodium, eplerenone, renin inhibitor and statins. The average follow-up of all clinical trials was 18.75 (s.d.: 13.25) months with a range from 2 to 48 months.

\section{Changes in pulse wave velocity}

Overall and stratified changes in PWV between ARB treatment and control groups are provided in Figure 2 and Table 3. PWV is a validated method to quantify arterial stiffness, and it can be measured in different arterial segments. baPWV and cfPWV are the most common indices. We separately analyzed changes in brachial-ankle PWV (baPWV) and carotid-femoral PWV (cfPWV) in this metaanalysis. Overall reductions in cfPWV and baPWV were statistically significant by an average of $-42.52 \mathrm{~cm} \mathrm{~s}^{-1}$ (95\% CI: -81.82 to -3.21 ; $P=0.034)$ and $-107.08 \mathrm{~cm} \mathrm{~s}^{-1}(95 \%$ CI: -133.98 to -80.18 ; $P<0.0005$ ), respectively, after treatment with ARBs (Figure 2). There was strong evidence of heterogeneity in both comparisons. Overall reductions were strengthened for cfPWV and baPWV by -49.03 $\mathrm{cm} \mathrm{s}^{-1}$ (95\% CI: -92.78 to $\left.-5.28 ; P=0.028\right)$ and $-121.17 \mathrm{~cm} \mathrm{~s}^{-1}$ (95\% CI: -151.39 to $-90.96 ; P<0.0005)$, respectively, after the removal of clinical trials that included controls who received ACEI or renin inhibitors, which might have exerted an effect similar to ARBs.

Stratified analyses by the type of ARBs detected significant reductions in baPWV for telmisartan $\left(\mathrm{WMD}=-110.82 \mathrm{~cm} \mathrm{~s}^{-1}\right.$; 95\% CI: -145.66 to $-55.98 ; P<0.0005)$ and valsartan $(\mathrm{WMD}=$ $-104.59 \mathrm{~cm} \mathrm{~s}^{-1}$; $95 \% \mathrm{CI}:-154.01$ to $\left.-55.18 ; P<0.0005\right)$, and reductions in cfPWV for valsartan $(\mathrm{WMD}=-65.58 \mathrm{~cm} / \mathrm{s} ; 95 \% \mathrm{CI}$ : -124.84 to $-6.32 ; P=0.030$ ) with evident heterogeneity (Table 3 ). Stratification by the type of drugs taken by controls revealed a significant reduction in cfPWV for placebo $\left(\mathrm{WMD}=-79.65 \mathrm{~cm} \mathrm{~s}^{-1}\right.$; $P=0.001 ; I^{2}=70.6 \%$ ), and baPWV was significantly reduced for comparisons of ARBs with diuretics $\left(\mathrm{WMD}=-77.83 \mathrm{~cm} \mathrm{~s}^{-1} ; P<0.0005\right)$, calcium channel blockers (WMD $\left.=-130.74 \mathrm{~cm} \mathrm{~s}^{-1} ; P<0.0005\right)$ and non-RAS drugs (WMD $=-81.66 \mathrm{~cm} \mathrm{~s}^{-1} ; P<0.001$ ) (Figure 2).

\section{Changes in blood pressure and heart rate}

Overall and stratified changes in blood pressure and heart rate between the ARB treatment and control groups are provided in Tables 4 and 5. Overall changes in SBP, diastolic blood pressure, pulse pressure and heart rate were not obvious between the two groups, and there was strong evidence of heterogeneity. Moreover, changes in PWV regressed with changes in pulse pressure in the ARB treatment group, and there was no observable significance for either $\operatorname{cfPWV}(P=0.229)$ or baPWV $(P=0.228)$, even after adjusting for age, gender, body mass index and treatment duration.

The grouping of studies by ARB type revealed an obvious reduction in SBP for telmisartan (WMD $=-1.12 \mathrm{~mm} \mathrm{Hg}$; 95\% CI: -1.82 to 
$-0.42 ; P=0.002 ; I^{2}=0.0 \%$ ) (Table 4) but a significant increase in heart rate for losartan (WMD $=6.66$ beats per minute; $95 \%$ CI: 0.77 to $12.55 ; P=0.027$ ) (Table 5). Subgroup analysis by the type of drugs taken by controls revealed significant reductions in SBP for comparisons of ARBs with placebo $(\mathrm{WMD}=-3.81 \mathrm{~mm} \mathrm{Hg}$; 95\% CI: -6.70 to $\left.-0.91 ; P=0.010 ; I^{2}=77.2 \%\right)$ and diuretics $(\mathrm{WMD}=-1.31 \mathrm{~mm} \mathrm{Hg} ; 95 \% \mathrm{CI}:-2.33$ to $-0.27 ; \quad P=0.013$; $I^{2}=0.0 \%$ ), and reductions in diastolic blood pressure for placebo

Table 1 The baseline characteristics of all eligible trials in this meta-analysis

\begin{tabular}{|c|c|c|c|c|c|c|c|c|c|c|}
\hline Author (year) & Ethnicity & Status & PWV type & Design & Masking & ARB type & Drugs in controls & Treatment & Control & Follow-up (months) \\
\hline Mahmud and Feely 8 & Caucasian & Hypertension & cfPWV & Crossover & Open & Valsartan & ACEI & 12 & 12 & 4 \\
\hline Mahmud and Feely 8 & Caucasian & Hypertension & cfPWV & Crossover & Double & Losartan & Diuretics & 11 & 11 & 4 \\
\hline Asmar et al. ${ }^{26}$ & Caucasian & Hypertension & cfPWV & Crossover & Double & Telmisartan & Placebo & 20 & 20 & 3 \\
\hline Suzuki et al. ${ }^{36}$ & East Asian & Others & baPWV & Parallel & Double & Valsartan & Placebo & 14 & 10 & 48 \\
\hline Takami and Shigemasa ${ }^{37}$ & East Asian & Hypertension & baPWV & Parallel & NA & Valsartan & $\mathrm{CCB}$ & 20 & 16 & 12 \\
\hline Takami and Shigemasa ${ }^{37}$ & East Asian & Hypertension & baPWV & Parallel & NA & Valsartan & $\mathrm{CCB}$ & 20 & 20 & 12 \\
\hline Takami and Shigemasa ${ }^{37}$ & East Asian & Hypertension & baPWV & Parallel & NA & Valsartan & ACEI & 20 & 20 & 12 \\
\hline Munakata et al. ${ }^{32}$ & East Asian & Hypertension & baPWV & Parallel & NA & Valsartan & $\mathrm{CCB}$ & 21 & 20 & 12 \\
\hline Anan 25 & East Asian & Hypertension & baPWV & Parallel & NA & Valsartan & ACEI & 10 & 11 & 40 \\
\hline Ichihara et al. ${ }^{27}$ & East Asian & Hypertension & baPWV & Parallel & Open & Losartan & ACEI & 22 & 21 & 48 \\
\hline Ichihara et al. ${ }^{27}$ & East Asian & Hypertension & baPWV & Parallel & Open & Losartan & Placebo & 22 & 21 & 48 \\
\hline Ichihara et al. ${ }^{28}$ & East Asian & Hypertension & baPWV & Parallel & NA & Valsartan & $\mathrm{CCB}$ & 50 & 50 & 48 \\
\hline Rajagopalan et al. 34 & Caucasian & Healthy & cfPWV & Crossover & Double & Valsartan & Placebo & 33 & 33 & 13 \\
\hline Morimoto et al. ${ }^{31}$ & East Asian & Hypertension & baPWV & Parallel & Open & Telmisartan & CCB & 21 & 22 & 24 \\
\hline Zhao ${ }^{50}$ & East Asian & Hypertension & cfPWV & Parallel & NA & Valsartan & Placebo & 20 & 20 & 12 \\
\hline Rehman et al. ${ }^{35}$ & Caucasian & Hypertension & cfPWV & Parallel & Double & Losartan & ACEI & 19 & 20 & 12 \\
\hline Nakayama et al. ${ }^{33}$ & East Asian & Hypertension & baPWV & Parallel & Double & Telmisartan & Beraprost sodium & 20 & 20 & 12 \\
\hline Kosch et al. ${ }^{29}$ & Caucasian & Hypertension & cfPWV & Parallel & Double & Valsartan & Beta blocker & 25 & 27 & 12 \\
\hline Zheng and Lin ${ }^{51}$ & East Asian & Hypertension & baPWV & Parallel & Open & Valsartan & Diuretics & 21 & 18 & 12 \\
\hline Zheng and Lin ${ }^{51}$ & East Asian & Hypertension & baPWV & Parallel & Open & Valsartan & Beta blocker & 21 & 20 & 12 \\
\hline Zheng and $\operatorname{Lin}^{51}$ & East Asian & Hypertension & baPWV & Parallel & Open & Valsartan & $\mathrm{CCB}$ & 21 & 24 & 12 \\
\hline Zheng and $\operatorname{Lin}^{51}$ & East Asian & Hypertension & baPWV & Parallel & NA & Valsartan & ACEI & 21 & 22 & 12 \\
\hline Nakamura et al. ${ }^{12}$ & East Asian & Hypertension & baPWV & Parallel & Open & Telmisartan & $\mathrm{CCB}$ & 15 & 15 & 48 \\
\hline Ishii et al. ${ }^{17}$ & East Asian & Hypertension & baPWV & Parallel & Open & Candesartan & $\mathrm{CCB}$ & 11 & 11 & 12 \\
\hline Shigenaga et al.22 & East Asian & Hypertension & baPWV & Parallel & NA & Valsartan & Non-RAS & 15 & 15 & 24 \\
\hline Shigenaga et al. ${ }^{22}$ & East Asian & Hypertension & baPWV & Parallel & NA & Candesartan & Non-RAS & 15 & 15 & 24 \\
\hline Ruan et al. ${ }^{46}$ & East Asian & heart failure & baPWV & Parallel & Open & Irbesartan & Non-RAS & 25 & 22 & 12 \\
\hline Li et al. ${ }^{43}$ & East Asian & Hypertension & baPWV & Parallel & Open & Telmisartan & ACEI & 34 & 34 & 12 \\
\hline Li et al. ${ }^{43}$ & East Asian & Hypertension & baPWV & Parallel & Open & Telmisartan & $\mathrm{CCB}$ & 34 & 34 & 12 \\
\hline Mitsuhashi et al. ${ }^{11}$ & East Asian & Hypertension & baPWV & Parallel & Open & Losartan & Non-RAS & 20 & 20 & 48 \\
\hline Long and Liu ${ }^{45}$ & East Asian & Others & baPWV & Parallel & NA & Valsartan & $\mathrm{CCB}$ & 35 & 40 & 24 \\
\hline $\mathrm{Li}$ and Wang44 & East Asian & Hypertension & baPWV & Parallel & Open & Telmisartan & $\mathrm{CCB}$ & 48 & 45 & 12 \\
\hline $\mathrm{Li}$ and Wang44 & East Asian & Hypertension & baPWV & Parallel & Open & Telmisartan & Diuretics & 48 & 42 & 12 \\
\hline Ruan et al. ${ }^{47}$ & East Asian & Hypertension & baPWV & Parallel & Open & Candesartan & Non-RAS & 23 & 19 & 12 \\
\hline Han et al. ${ }^{19}$ & East Asian & Others & baPWV & Parallel & Open & Valsartan & Statins & 57 & 57 & 24 \\
\hline Lunder et al.. ${ }^{21}$ & Caucasian & Healthy & cfPWV & Parallel & Double & Valsartan & Placebo & 20 & 20 & 4 \\
\hline Wu et al. ${ }^{48}$ & East Asian & Hypertension & baPWV & Parallel & NA & Valsartan & $\mathrm{CCB}$ & 48 & 50 & 2 \\
\hline Wu et al. ${ }^{48}$ & East Asian & Hypertension & baPWV & Parallel & NA & Valsartan & ACEI & 48 & 49 & 2 \\
\hline He et al..39 & East Asian & Hypertension & baPWV & Parallel & NA & Valsartan & $\mathrm{CCB}$ & 44 & 44 & 12 \\
\hline Feng 38 & East Asian & Hypertension & baPWV & Parallel & Open & Telmisartan & $\mathrm{CCB}$ & 44 & 49 & 12 \\
\hline Tomiyama et al. ${ }^{20}$ & East Asian & Hypertension & baPWV & Parallel & Open & Candesartan & $\mathrm{CCB}$ & 56 & 57 & 32 \\
\hline Hayoz et al. ${ }^{10}$ & Caucasian & Hypertension & baPWV & Parallel & Double & Valsartan & $\mathrm{CCB}$ & 63 & 62 & 38 \\
\hline Spanos et al. ${ }^{13}$ & Caucasian & Hypertension & cfPWV & Parallel & NA & Valsartan & Renin inhibitor & 14 & 15 & 24 \\
\hline Yang et al. ${ }^{49}$ & East Asian & Hypertension & baPWV & Parallel & NA & Valsartan & Diuretics & 42 & 40 & 24 \\
\hline Kong et al.. ${ }^{41}$ & East Asian & Hypertension & baPWV & Parallel & Open & Irbesartan & ACEI & 34 & 34 & 12 \\
\hline Kong et al. ${ }^{41}$ & East Asian & Hypertension & baPWV & Parallel & Open & Irbesartan & $\mathrm{CCB}$ & 34 & 34 & 12 \\
\hline $\mathrm{Li}$ and $\mathrm{Ma}^{42}$ & East Asian & Hypertension & baPWV & Parallel & Open & Telmisartan & $\mathrm{CCB}$ & 33 & 34 & 24 \\
\hline Huang et al. ${ }^{40}$ & East Asian & Hypertension & baPWV & Parallel & Double & Losartan & Eplerenone & 40 & 37 & 12 \\
\hline Kim et al. ${ }^{11}$ & East Asian & Hypertension & cfPWV & Parallel & Open & Losartan & Beta blocker & 88 & 94 & 24 \\
\hline Ihm et al. ${ }^{24}$ & East Asian & Hypertension & cfPWV & Parallel & Open & Losartan & $\mathrm{CCB}$ & 99 & 101 & 24 \\
\hline Agnoletti et al. ${ }^{23}$ & Caucasian & Hypertension & cfPWV & Parallel & Open & Candesartan & $\mathrm{CCB}$ & 33 & 33 & 12 \\
\hline Agnoletti et al. ${ }^{23}$ & Caucasian & Hypertension & cfPWV & Parallel & Open & Candesartan & Diuretics & 33 & 44 & 12 \\
\hline Agnoletti et al. ${ }^{23}$ & Caucasian & Hypertension & cfPWV & Parallel & Open & Candesartan & Placebo & 33 & 35 & 12 \\
\hline
\end{tabular}


Table 2 The baseline characteristics of all study patients between the treatment and control groups in this meta-analysis

\begin{tabular}{|c|c|c|c|c|c|c|c|c|c|c|}
\hline \multirow[b]{2}{*}{ Author } & \multicolumn{2}{|c|}{ Age (years) } & \multicolumn{2}{|c|}{ Gender (male, \%) } & \multicolumn{2}{|c|}{$B M I\left(k g m^{-2}\right)$} & \multicolumn{2}{|c|}{$P W V$ in treatment group } & \multicolumn{2}{|c|}{ PWV in control group } \\
\hline & Treatment & Control & Treatment & Control & Treatment & Control & Baseline & Follow-up & Baseline & Follow-up \\
\hline Mahmud and Feely ${ }^{8}$ & 49 & 49 & NA & NA & NA & NA & 1110 & 1050 & 1110 & 1040 \\
\hline Mahmud and Feely ${ }^{8}$ & 57 & 57 & 45.5 & 45.5 & NA & NA & 1175 & 1035 & 1155 & 1170 \\
\hline Asmar et al..26 & NA & NA & NA & NA & NA & NA & 1253 & NA & 1307 & NA \\
\hline Suzuki H (2003) & 56 & 57 & NA & NA & NA & NA & 1700 & 1470 & 1670 & 1440 \\
\hline Takami and Shigemasa ${ }^{37}$ & 71 & 72.8 & 100 & 100 & 22.8 & 23.2 & NA & NA & NA & NA \\
\hline Takami and Shigemasa ${ }^{37}$ & 71 & 72 & 100 & 100 & 22.8 & 22.7 & NA & NA & NA & NA \\
\hline Takami and Shigemasa ${ }^{37}$ & 71 & 71.4 & 100 & 100 & 22.8 & 22.9 & NA & NA & NA & NA \\
\hline Munakata et al. ${ }^{32}$ & 53 & 55 & 47.6 & 50 & 24.8 & 25.3 & 1669 & NA & 1622 & NA \\
\hline Anan et al. ${ }^{25}$ & 59 & 59 & 10 & 54.5 & 25.5 & 25.4 & 1853 & 1615 & 1818 & 1613 \\
\hline Ichihara et al. ${ }^{27}$ & 65 & 61 & 59.1 & 52.4 & 20.8 & 20.1 & 2004 & 1898 & 2012 & 1870 \\
\hline Ichihara et al. ${ }^{27}$ & 65 & 63 & 59.1 & 57.1 & 20.8 & 20.8 & 2004 & 1898 & 2008 & 2144 \\
\hline Ichihara et al. ${ }^{28}$ & 54.3 & 53.9 & 72 & 76 & 24.2 & 24.6 & 1671 & 1489 & 1723 & 1517 \\
\hline Rajagopalan et al. ${ }^{34}$ & 71 & 71 & 60 & 60 & NA & NA & 787 & 730 & 789 & 786 \\
\hline Morimoto et al. ${ }^{31}$ & 56 & 58 & 43 & 41 & 24.8 & 24 & 1699 & 1432 & 1611 & 1540 \\
\hline Zhao 50 & 66.5 & 66.5 & 62.5 & 62.5 & NA & NA & 1280 & 800 & 1300 & 1128 \\
\hline Rehman et al. ${ }^{35}$ & 52.5 & 53.1 & NA & NA & 26.6 & 27.7 & 1200 & NA & 1100 & NA \\
\hline Nakayama et al. ${ }^{33}$ & 71.7 & 70.7 & 60 & 60 & 23.4 & 21.9 & 1985 & NA & 2011 & NA \\
\hline Kosch et al. ${ }^{29}$ & 45.4 & 46.2 & 51.4 & 57.6 & 29 & 28.2 & 1090 & 1030 & 1100 & 1040 \\
\hline Zheng and $\operatorname{Lin}^{51}$ & 52 & 50 & 61.9 & 55.6 & 24.8 & 24.7 & 1090 & 842.6 & 1060 & 967.8 \\
\hline Zheng and $\operatorname{Lin}^{51}$ & 52 & 51 & 61.9 & 60 & 24.8 & 24.2 & 1090 & 842.6 & 1120 & 945.3 \\
\hline Zheng and $\operatorname{Lin}^{51}$ & 52 & 52 & 61.9 & 62.5 & 24.8 & 25.1 & 1090 & 842.6 & 1080 & 706.3 \\
\hline Zheng and $\operatorname{Lin}^{51}$ & 52 & 50 & 61.9 & 54.5 & 24.8 & 24.5 & 1090 & 842.6 & 1110 & 868 \\
\hline Nakamura et al. ${ }^{12}$ & 45 & 47 & 60 & 60 & 25.4 & 24.8 & 1680 & 1460 & 1620 & 1660 \\
\hline Ishii et al. ${ }^{17}$ & 68.4 & 68 & NA & NA & 23.9 & 23.5 & 2007.8 & 1364 & 1985.7 & 1813 \\
\hline Shigenaga et al. ${ }^{22}$ & 53.1 & 53.3 & 66.7 & 73.3 & 25.1 & 25.8 & 1953 & 1820 & 1981 & 1888 \\
\hline Shigenaga et al. ${ }^{22}$ & 52.9 & 53.3 & 60 & 73.3 & 25.1 & 25.8 & 1985 & 1837 & 1981 & 1888 \\
\hline Ruan et al. ${ }^{46}$ & 64.8 & 63.5 & 68 & 59 & NA & NA & 1716.5 & 1482.7 & 1731.6 & 1610.4 \\
\hline Li et al. ${ }^{43}$ & 58.3 & 58 & 64.7 & 61.8 & NA & NA & 1859 & 1566 & 1859 & 1702 \\
\hline $\mathrm{Li}$ et al. ${ }^{43}$ & 58.3 & 57.1 & 64.7 & 67.6 & NA & NA & 1859 & 1566 & 1780 & 1559 \\
\hline Mitsuhashi et al. ${ }^{11}$ & 68.8 & 63.3 & 60 & 60 & 24.6 & 25.6 & 2011 & 1791 & 2008 & 1907 \\
\hline Long and Liư ${ }^{45}$ & 59.3 & 57.4 & 65.7 & 65 & 28.9 & 24.9 & 1260 & 1080 & 1210 & 1140 \\
\hline $\mathrm{Li}$ and Wang ${ }^{44}$ & 72 & 73 & NA & NA & NA & NA & 2177 & 2085 & 2195 & 2117 \\
\hline $\mathrm{Li}$ and Wang ${ }^{44}$ & 72 & 74 & NA & NA & NA & NA & 2177 & 2085 & 2186 & 2174 \\
\hline Ruan et al. ${ }^{47}$ & 64.8 & 63.5 & 69 & 63 & NA & NA & 1764.4 & 1524.3 & 1759.7 & 1634.5 \\
\hline Han et al. ${ }^{19}$ & 48.8 & 48.8 & 51.2 & 45.9 & 22.8 & 22.8 & 1691.5 & 1635 & 1617 & 1528.9 \\
\hline Lunder et al. ${ }^{21}$ & 42.8 & 43.1 & 100 & 100 & 25.9 & 26.6 & 582 & 519 & 577 & 580 \\
\hline Wu et al. ${ }^{48}$ & NA & NA & 77.1 & 76 & NA & NA & 1797.8 & 1027.8 & 1850.6 & 1411.6 \\
\hline Wu et al. ${ }^{48}$ & NA & NA & 77.1 & 79.6 & NA & NA & 1797.8 & 1027.8 & 1870.8 & 1170.8 \\
\hline He et al. 39 & 60.5 & 59.4 & 0 & 0 & 24.5 & 25.4 & 2007.8 & 1370.5 & 1985.7 & 1812.7 \\
\hline Feng 38 & 57.2 & 56.3 & 61 & 61 & NA & NA & 1829.5 & 1464.8 & 1875.6 & 1578.4 \\
\hline Tomiyama et al..20 & 56 & 58 & 64.2 & 63.1 & 24.1 & 23.5 & 1741 & 1576 & 1774 & 1662 \\
\hline Hayoz et al. ${ }^{10}$ & 62.3 & 60.4 & 0 & 0 & 27.5 & 27 & NA & NA & NA & NA \\
\hline Spanos et al. ${ }^{13}$ & 60 & 55 & 71.4 & 66.7 & 27.5 & 29.6 & 1110 & 1000 & 940 & 950 \\
\hline Yang et al. ${ }^{49}$ & 57.3 & 56.8 & 69 & 67.5 & 24.5 & 24.4 & 1230 & 1090 & 1250 & 1180 \\
\hline Kong et al. ${ }^{41}$ & 52.4 & 52.2 & 64.7 & 67.6 & NA & NA & 1864 & 1553 & 1856 & 1623 \\
\hline Kong et al. ${ }^{41}$ & 52.4 & 52 & 64.7 & 61.8 & NA & NA & 1864 & 1553 & 1862 & 1562 \\
\hline $\mathrm{Li}$ and $\mathrm{Ma}^{42}$ & 58.6 & 58.1 & 54 & 58 & NA & NA & 1763.5 & 1436.5 & 1722.7 & 1465.7 \\
\hline Huang et al. ${ }^{40}$ & 49.2 & 48.6 & NA & NA & NA & NA & 1718.9 & 1610.1 & 1648.1 & 1570.7 \\
\hline Kim et al. ${ }^{11}$ & 48.7 & 50.5 & 62.5 & 56.4 & 26 & 26 & 752 & 780 & 768 & 756 \\
\hline $\mathrm{Ihm}$ et al. ${ }^{24}$ & 50.6 & 51.1 & 61.6 & 54.5 & 25.1 & 25.4 & 900 & 890 & 850 & 860 \\
\hline Agnoletti et al. ${ }^{23}$ & 57.2 & 59.5 & 55 & 52 & NA & NA & 960 & 970 & 1030 & 980 \\
\hline Agnoletti et al..23 & 57.2 & 59.2 & 55 & 57 & NA & NA & 960 & 970 & 970 & 980 \\
\hline Agnoletti et al..23 & 57.2 & 56.6 & 55 & 46 & NA & NA & 960 & 970 & 970 & 960 \\
\hline
\end{tabular}

Abbreviations: BMI, body mass index; NA, not available; PWV, pulse wave velocity. 


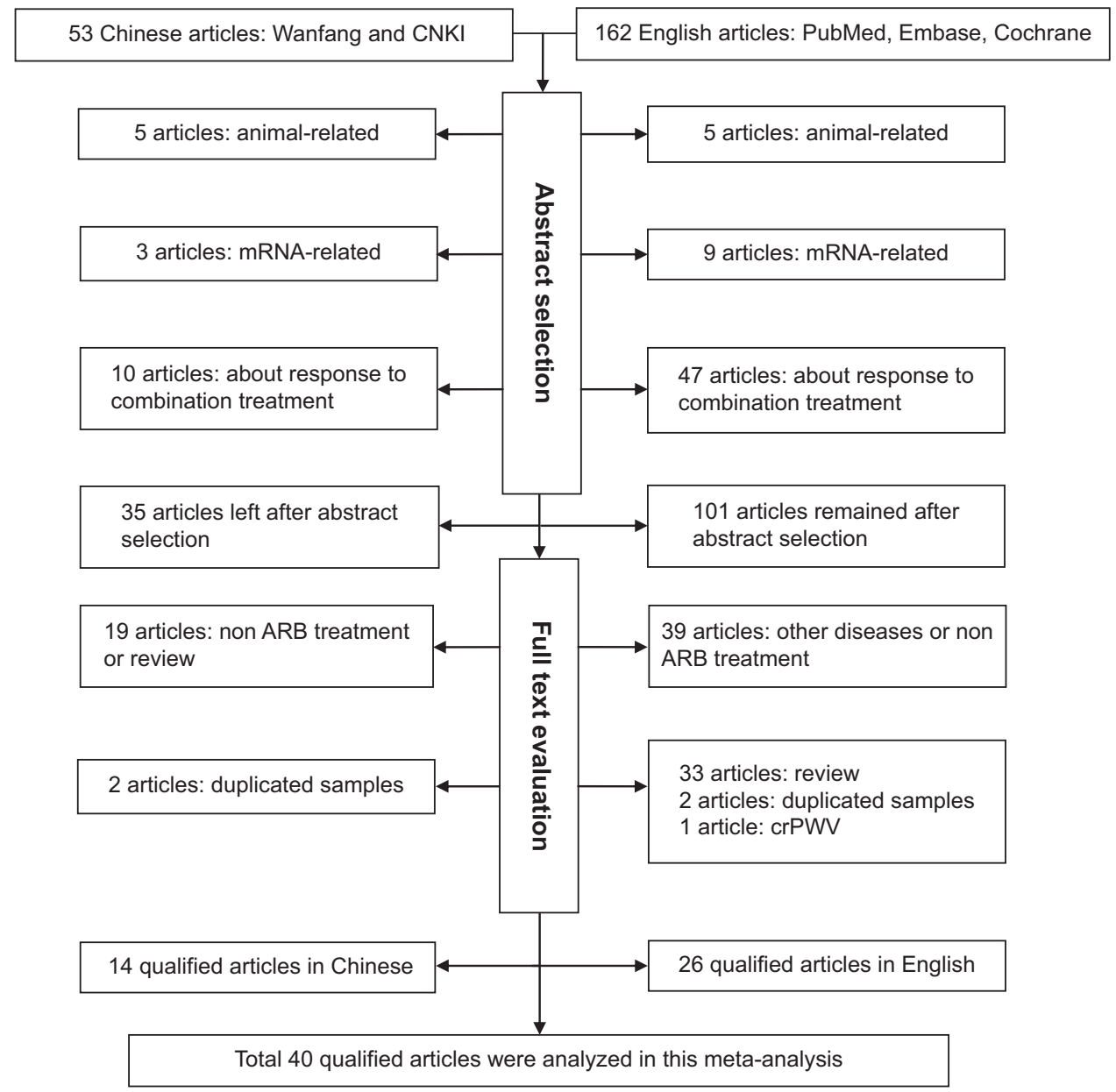

Figure 1 Flow diagram of search strategy and trial selection.
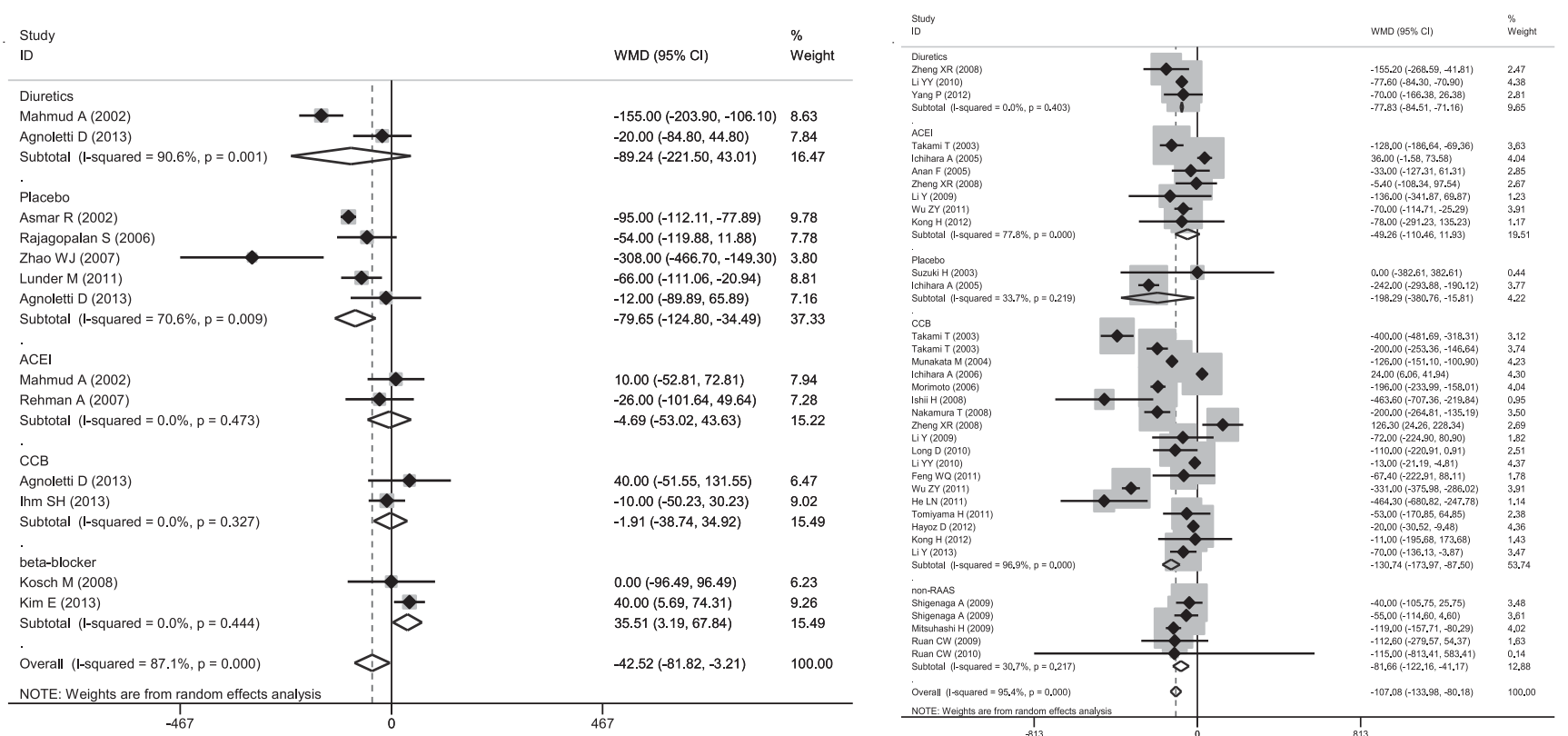

Figure 2 Forest plots of carotid-femoral and brachial-ankle pulse wave velocity (left and right) changes between angiotensin receptor blocker treatment and control groups according to the types of drugs used in the controls. 
Table 3 Weighted mean differences in pulse wave velocity between the ARB treatment and control groups according to the type of drugs in treatment group

\begin{tabular}{|c|c|c|c|}
\hline Subgroups & Studies ( $n$ ) & $W M D ; 95 \% \mathrm{Cl} ; \mathrm{P}$ & $1^{2}(\mathrm{P})$ \\
\hline \multicolumn{4}{|l|}{ baPWV } \\
\hline Telmisartan & 9 & $-100.82 ;-145.66$ to $-55.98 ; 0.000$ & $96.3 \%(0.000)$ \\
\hline Valsartan & 19 & $-104.59 ;-154.01$ to $-55.18 ; 0.000$ & $95.7 \%(0.000)$ \\
\hline Candesartan & 4 & $-139.49 ;-288.74$ to $9.76 ; 0.067$ & $71.0 \%(0.016)$ \\
\hline Irbesartan & 3 & $-69.70 ;-176.80$ to $37.40 ; 0.202$ & $0.0 \%(0.723)$ \\
\hline Losartan & 4 & $37.02 ;-122.88$ to $48.86 ; 0.398$ & $92.7 \%(0.000)$ \\
\hline Valsartan & 6 & $-65.58 ;-124.84$ to $-6.32 ; 0.030$ & $69.0 \%(0.006)$ \\
\hline Candesartan & 3 & $-3.77 ;-47.53$ to $39.99 ; 0.866$ & $0.0 \%(0.559)$ \\
\hline
\end{tabular}

Abbreviations: $95 \% \mathrm{Cl}, 95 \%$ confidence interval; ARB, angiotensin receptor blocker; baPWV and cfPWV, brachial-ankle and carotid-femoral pulse wave velocity; $R$, inconsistency index; WMD, weighted mean difference.

Table 4 Weighted mean differences in systolic and diastolic blood pressure between the ARB treatment and control groups according to the types of drugs taken in both groups

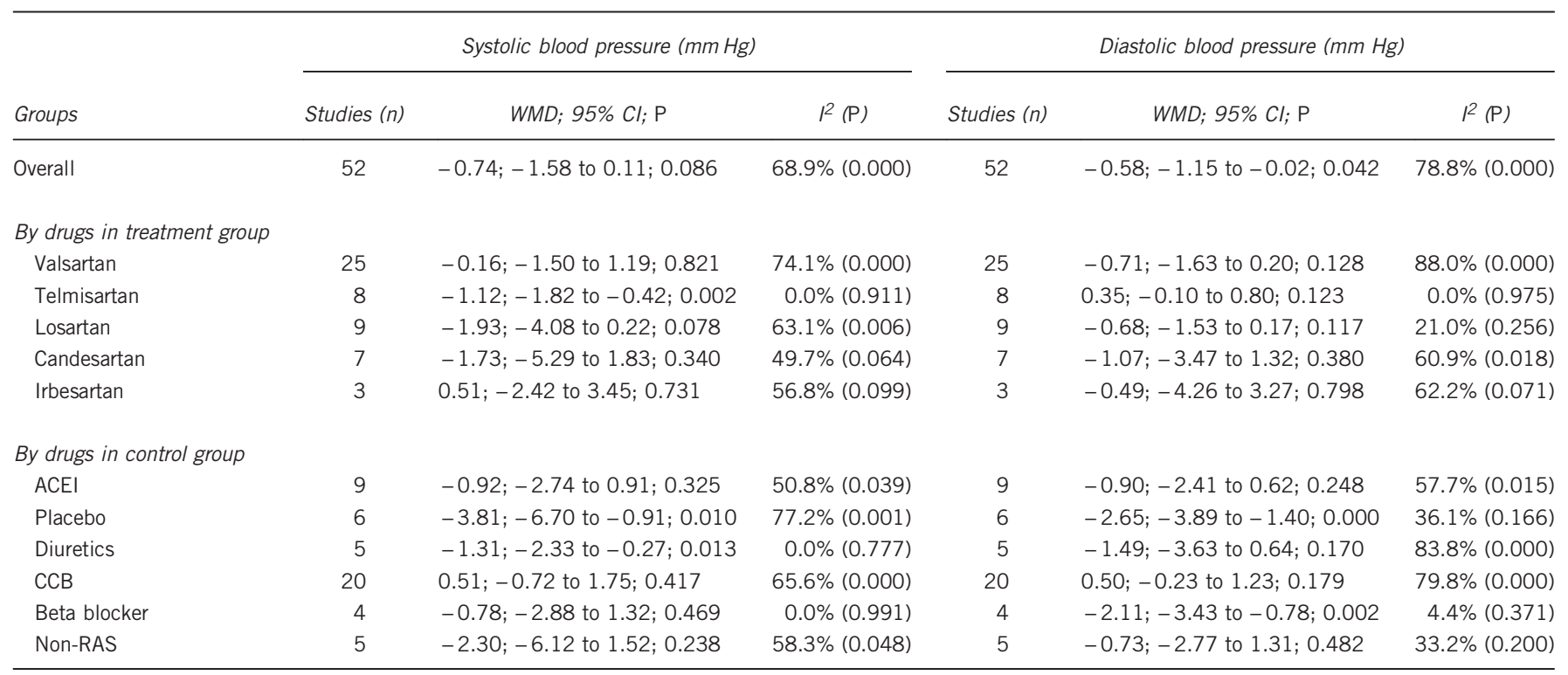

Abbreviations: $95 \% \mathrm{Cl}, 95 \%$ confidence interval; ACEI, angiotensin converting enzyme inhibitor; ARB, angiotensin receptor blocker; CCB, calcium channel blocker; $R$, inconsistency index; NA, not available; RAS, renin-angiotensin system; WMD, weighted mean difference.

$(\mathrm{WMD}=-2.65 \mathrm{~mm} \mathrm{Hg} ; \quad 95 \% \quad \mathrm{CI}:-3.89$ to $-1.40 ; \quad P<0.0005$; $\left.I^{2}=36.1 \%\right)$ and beta blockers (WMD $=-2.11 \mathrm{~mm} \mathrm{Hg} ; 95 \%$ CI: -3.43 to $\left.-0.78 ; P=0.002 ; I^{2}=4.4 \%\right)$, with improved heterogeneity (Table 4). In contrast, comparisons of ARBs with beta blockers revealed a significant increase in heart rate $(\mathrm{WMD}=8.47$ beats per minute; 95\% CI: $0.87-16.08 ; \quad P=0.029 ; \quad I^{2}=87.1 \%$ ) (Table 5).

\section{Publication bias}

Publication bias was only calculated for overall estimates because of the small number of studies involved in subgroup analyses. There was a low probability of publication bias for the overall comparisons of baPWV and cfPWV between the ARB treatment and control groups, as reflected by Begg's test ( $P=0.479$ and 0.631 , respectively). Similarly, there was no observable publication bias for the overall comparisons of SBP ( $P$ for Begg's test: 0.877$)$, diastolic blood pressure $(P=0.350)$, pulse pressure $(P=0.921)$ and heart rate $(P=0.718)$ between the two groups.

\section{Meta-regression analysis}

We performed meta-regression analyses to assess the confounding impact of age, gender, body mass index and treatment duration on the treatment of ARBs versus other types of drugs in terms of PWV and other related indexes and further explore potential sources of heterogeneity. Age explained some part of the heterogeneity for baPWV (regression coefficient: $-5.18 ; P=0.028$ ) but not $\mathrm{cfPWV}$ (regression coefficient: $-2.87 ; P=0.300$ ), which indicates that the change in PWV was negatively associated with increases in age. No significance was observed for the other confounders.

\section{DISCUSSION}

The key finding of this study was that ARBs, particularly telmisartan and valsartan, significantly reduced baPWV and cfPWV relative to placebo, except SBP. To the authors' knowledge, this study is the most comprehensive meta-analysis to assess the comparison of ARBs with the other types of drugs in terms of PWV and related indices of arterial stiffness. 
Table 5 Weighted mean differences in pulse pressure and heart rate between the ARB treatment and control groups according to the types of drugs taken in both groups

\begin{tabular}{|c|c|c|c|c|c|c|}
\hline \multirow[b]{2}{*}{ Groups } & \multicolumn{3}{|c|}{ Pulse pressure ( $\mathrm{mm} \mathrm{Hg}$ ) } & \multicolumn{3}{|c|}{ Heart rate (beats per minute) } \\
\hline & Studies (n) & WMD; $95 \% \mathrm{Cl} ; \mathrm{P}$ & $R^{2}(\mathrm{P})$ & Studies (n) & WMD; 95\% Cl; P & $R^{2}(\mathrm{P})$ \\
\hline Overall & 28 & $-0.45 ;-1.41$ to $0.51 ; 0.359$ & $44.0 \%(0.007)$ & 17 & $1.34 ;-0.57$ to $3.26 ; 0.169$ & $74.7 \%(0.000)$ \\
\hline \multicolumn{7}{|c|}{ By drugs in treatment group } \\
\hline Valsartan & 13 & $-0.58 ;-2.26$ to $1.10 ; 0.501$ & $70.2 \%(0.000)$ & 8 & $0.94 ;-0.30$ to $2.18 ; 0.138$ & $12.2 \%(0.335)$ \\
\hline Telmisartan & 4 & $-0.10 ;-2.35$ to $2.16 ; 0.934$ & $0.0 \%(0.706)$ & 2 & $0.98 ;-3.62$ to $5.58 ; 0.675$ & $0.0 \%(0.766)$ \\
\hline Losartan & 3 & $0.45 ;-2.35$ to $3.26 ; 0.751$ & $22.9 \% 90.273)$ & 4 & $6.66 ; 0.77$ to $12.55 ; 0.027$ & $89.8 \%(0.000)$ \\
\hline Candesartan & 5 & $-0.36 ;-2.87$ to $2.16 ; 0.782$ & $0.0 \% 90.603)$ & 3 & $-2.81 ;-5.85$ to $0.23 ; 0.070$ & $0.0 \%(0.755)$ \\
\hline Irbesartan & 3 & $-1.27 ;-3.46$ to $0.93 ; 0.257$ & $0.0 \%(0.866)$ & & & \\
\hline \multicolumn{7}{|c|}{ By drugs in control group } \\
\hline ACEI & 6 & $-1.27 ;-3.17$ to $0.63 ; 0.189$ & $0.0 \%(0.516)$ & 4 & $0.41 ;-1.51$ to $2.32 ; 0.675$ & $0.0 \%(0.786)$ \\
\hline Placebo & 3 & $-2.88 ;-6.40$ to $0.64 ; 0.109$ & $0.0 \%(0.869)$ & & & \\
\hline Diuretics & 2 & $0.95 ;-2.62$ to $4.52 ; 0.603$ & $0.0 \%(0.432)$ & & & \\
\hline CCB & 12 & $-0.45 ;-1.91$ to $1.0 ; 0.540$ & $68.8 \%(0.000)$ & 7 & $1.00 ;-0.39$ to $2.39 ; 0.159$ & $20.8 \%(0.271)$ \\
\hline Beta blocker & & & & 3 & $8.47 ; 0.87$ to $16.08 ; 0.029$ & $87.1 \%(0.000)$ \\
\hline Non-RAS & 4 & $0.77 ;-1.93$ to $3.47 ; 0.574$ & $0.0 \%(0.563)$ & & & \\
\hline
\end{tabular}

Abbreviations: $95 \% \mathrm{Cl}, 95 \%$ confidence interval; ACEI, angiotensin converting enzyme inhibitor; ARB, angiotensin receptor blocker; CCB, calcium channel blocker; $P^{2}$, inconsistency index; RAS, renin-angiotensin system; NA, not available; WMD, weighted mean difference.

Angiotensin II is the terminal pressor effector molecular of RAS that plays an active role in blood pressure regulation via the induction of vasoconstriction and sodium and fluid retention and the initiation and progression of endothelial dysfunction and vascular remodeling. ${ }^{52}$ Therefore, it is reasonably expected that ARBs may improve arterial compliance via blockade of the negative role of angiotensin II in the endothelium, as exemplified by the improvement in vascular compliance and endothelial function in healthy elderly subjects taking ARBs. ${ }^{34}$ However, many, but not all, clinical studies documented the beneficial impact of ARBs on arterial stiffness. For example, Suzuki et al. ${ }^{36}$ observed a comparative reduction in PWV between treatment with and without ARBs. As a caveat, this lack of reproducibility might be attributed to the diverse types of drugs taken by controls or the individually underpowered clinical trials. We performed a metaanalysis of data from 40 articles and 3309 subjects and demonstrated significantly lower levels of baPWV and cfPWV and SBP in patients taking ARBs, especially telmisartan and valsartan, than placebo, which reinforces the selection of $\mathrm{ARBs}$ as the first-line treatment for arterial destiffening in clinical routine. Moreover, Shahin and coworkers summarized the impact of ACEIs on arterial stiffness and found that ACEIs significantly reduced PWV relative to the placebo, but this difference was non-significant relative to other antihypertensive agents, including ARBs, calcium channel blockers, beta blockers and diuretics. $^{53}$ Consistent with the results of this meta-analysis, the impact of ARBs on improvements in arterial elasticity was superior, but insignificant, compared with ACEIs. It is also worth stressing that the modulatory impact of ARBs on arterial stiffness is independent of the resulting blood pressure decrease, which was confirmed by many previous studies. ${ }^{8}$ These results suggest that additional mechanisms are invoked by ARBs in the treatment of arterial stiffness.

Several possible limitations should be acknowledged. First, a set of subgroup analyses was undertaken, but significant heterogeneity persisted in some subgroups. Second, we must have some reservations regarding the interpretation of our subgroup results because of the limited number of clinical trials with relatively small sample sizes in some subgroups. In fact, Hannah and colleagues suggested that the study power is low if the number of studies included in a metaanalysis is 10 or fewer. ${ }^{54}$ Third, our statistical tests reported a low probability of publication bias, which is consistent with all metaanalyses, but selection bias cannot be completely excluded because we only retrieved published articles and trials written in English or Chinese. Therefore, our conclusions require further verification in larger, more targeted clinical trials.

Taken together, meta-analyses of the data from 40 articles and 3309 subjects support an important role of ARB treatment in improving arterial stiffness. For practical reasons, successful validation of the present results with accumulating data from large randomized clinical trials will shed more light on the widespread administration of ARBs in the treatment of arterial stiffness in daily clinical practice.

\section{CONFLICT OF INTEREST}

The authors declare no conflict of interest.

\section{ACKNOWLEDGEMENTS}

We thank for the support provided by The Natural Science Foundation of Heilongjiang Province (Grant No. C201320).

1 Vlachopoulos C, Aznaouridis K, Stefanadis C. Prediction of cardiovascular events and all-cause mortality with arterial stiffness: a systematic review and meta-analysis. J Am Coll Cardiol 2010; 55: 1318-1327.

2 Otsuka K, Fukuda S, Shimada K, Suzuki K, Nakanishi K, Yoshiyama M, Yoshikawa J. Serial assessment of arterial stiffness by cardio-ankle vascular index for prediction of future cardiovascular events in patients with coronary artery disease. Hypertens Res 2014; 37: 1014-1020.

3 Seidlerova J, Filipovsky J, Mayer O, Wohlfahrt P, Cifkova R. Positive effects of antihypertensive treatment on aortic stiffness in the general population. Hypertens Res 2014; 37: 64-68.

4 Wolf G. Free radical production and angiotensin. Curr Hypertens Rep 2000; 2: 167-173.

5 Otsuka T, Munakata R, Kato K, Kodani E, Ibuki C, Kusama Y, Seino Y, Kawada T. Oscillometric measurement of brachial artery cross-sectional area and its relationship with cardiovascular risk factors and arterial stiffness in a middle-aged male population. Hypertens Res 2013; 36: 910-915. 
6 Shirai K, Utino J, Saiki A, Endo K, Ohira M, Nagayama D, Tatsuno I, Shimizu K, Takahashi M, Takahara A. Evaluation of blood pressure control using a new arteria stiffness parameter, cardio-ankle vascular index (CAVI). Curr Hypertens Rev 2013; 9: 66-75.

7 Kim EJ, Song WH, Lee JU, Shin MS, Lee S, Kim BO, Hong KS, Han SW, Park CG, Seo HS. Efficacy of losartan and carvedilol on central hemodynamics in hypertensives: a prospective, randomized, open, blinded end point, multicenter study. Hypertens Res 2014; 37: 50-56.

8 Mahmud A, Feely J. Effect of angiotensin ii receptor blockade on arterial stiffness: beyond blood pressure reduction. Am J Hypertens 2002; 15: 1092-1095.

$9 \mathrm{Kim}$ JH, Oh SJ, Lee JM, Hong EG, Yu JM, Han KA, Min KW, Son HS, Chang SA. The effect of an Angiotensin receptor blocker on arterial stiffness in type 2 diabetes mellitus patients with hypertension. Diabetes Metab J 2011; 35: 236-242.

10 Hayoz D, Zappe DH, Meyer MA, Baek I, Kandra A, Joly MP, Mazzolai L, Haesler E, Periard D. Changes in aortic pulse wave velocity in hypertensive postmenopausal women: comparison between a calcium channel blocker vs angiotensin receptor blocker regimen. J Clin Hypertens (Greenwich) 2012; 14: 773-778.

11 Kim EJ, Song WH, Lee JU, Shin MS, Lee S, Kim BO, Hong KS, Han SW, Park CG, Seo HS. Efficacy of losartan and carvedilol on central hemodynamics in hypertensives: a prospective, randomized, open, blinded end point, multicenter study. Hypertens Res 2013; 37: 50-6.

12 Nakamura T, Inoue T, Suzuki T, Kawagoe Y, Ueda Y, Koide H, Node K. Comparison of renal and vascular protective effects between telmisartan and amlodipine in hypertensive patients with chronic kidney disease with mild renal insufficiency. Hypertens Res 2008; 31: 841-850.

13 Spanos G, Kalaitzidis R, Karasavvidou D, Pappas K, Siamopoulos KC. Efficacy of aliskiren and valsartan in hypertensive patients with albuminuria: a randomized parallelgroup study. J Renin Angiotensin Aldosterone Syst 2012; 14: 315-21.

14 Moher D, Liberati A, Tetzlaff J, Altman DG. Preferred reporting items for systematic reviews and meta-analyses: the PRISMA statement. Ann Intern Med 2009; 151 264-269 W264.

15 DerSimonian R, Kacker R. Random-effects model for meta-analysis of clinical trials: an update. Contemp Clin Trials 2007; 28: 105-114.

16 Bowden J, Tierney JF, Copas AJ, Burdett S. Quantifying, displaying and accounting for heterogeneity in the meta-analysis of RCTs using standard and generalised Q statistics. BMC Med Res Methodol 2011; 11: 41.

17 Ishii H, Tsukada T, Yoshida M. Angiotensin II type-I receptor blocker, candesartan, improves brachial-ankle pulse wave velocity independent of its blood pressure lowerin effects in type 2 diabetes patients. Intern Med 2008; 47: 2013-2018.

18 Mitsuhashi H, Tamura K, Yamauchi J, Ozawa M, Yanagi M, Dejima T, Wakui H, Masuda S, Azuma K, Kanaoka T, Ohsawa M, Maeda A, Tsurumi-Ikeya Y, Okano Y, Ishigami T, Toya Y, Tokita Y, Ohnishi T, Umemura S. Effect of losartan on ambulatory short-term blood pressure variability and cardiovascular remodeling in hypertensive patients on hemodialysis. Atherosclerosis 2009; 207: 186-190.

19 Han SH, Kang EW, Yoon SJ, Yoon HS, Lee HC, Yoo TH, Choi KH, Han DS, Kang SW. Combined vascular effects of HMG-CoA reductase inhibitor and angiotensin recepto blocker in non-diabetic patients undergoing peritoneal dialysis. Nephrol Dial Transplant 2011; 26: 3722-3728.

20 Tomiyama H, Yoshida M, Yamada J, Matsumoto C, Odaira M, Shiina K, Yamashina A. Arterial-cardiac destiffening following long-term antihypertensive treatment. $\mathrm{Am} \mathrm{J}$ Hypertens 2011; 24: 1080-1086.

21 Lunder M, Janic M, Sabovic M. Reduction of age-associated arterial wall changes by low-dose valsartan. Eur J Prev Cardiol 2012; 19: 1243-1249.

22 Shigenaga A, Tamura K, Dejima T, Ozawa M, Wakui H, Masuda S, Azuma K, Tsurumi-Ikeya $Y$, Mitsuhashi H, Okano $Y$, Kokuho T, Sugano T, Ishigami T, Toya $Y$, Uchino K, Tokita Y, Umemura S. Effects of angiotensin II type 1 receptor blocker on blood pressure variability and cardiovascular remodeling in hypertensive patients on chronic peritoneal dialysis. Nephron Clin Pract 2009; 112: c31-40.

23 Agnoletti D, Zhang Y, Borghi C, Blacher J, Safar ME. Effects of antihypertensive drugs on central blood pressure in humans: a preliminary observation. Am J Hypertens 2013; 26: 1045-1052.

$24 \mathrm{lhm}$ SH, Jeon HK, Chae SC, Lim DS, Kim KS, Choi DJ, Ha JW, Kim DS, Kim KH, Cho MC, Baek SH. Belasco trial investigators RoK. Benidipine has effects similar to losartan on the central blood pressure and arterial stiffness in mild to moderate essential hypertension. Chin Med J (Engl) 2013; 126: 2021-2028.

25 Anan F, Takahashi N, Ooie T, Yufu K, Hara M, Nakagawa M, Yonemochi H, Saikawa T, Yoshimatsu $\mathrm{H}$. Effects of valsartan and perindopril combination therapy on left ventricular hypertrophy and aortic arterial stiffness in patients with essential hypertension. Eur J Clin Pharmacol 2005; 61: 353-359.

26 Asmar R, Gosse P, Topouchian J, N'Tela G, Dudley A, Shepherd GL. Effects of telmisartan on arterial stiffness in Type 2 diabetes patients with essential hypertension. $J$ Renin Angiotensin Aldosterone Syst 2002; 3: 176-180.

27 Ichihara A, Hayashi M, Kaneshiro Y, Takemitsu T, Homma K, Kanno Y, Yoshizawa M, Furukawa T, Takenaka T, Saruta T. Low doses of losartan and trandolapri improve arterial stiffness in hemodialysis patients. Am J Kidney Dis 2005; 45: 866-874
28 Ichihara A, Kaneshiro Y, Takemitsu T, Sakoda M. Effects of amlodipine and valsartan on vascular damage and ambulatory blood pressure in untreated hypertensive patients. J Hum Hypertens 2006; 20: 787-794.

29 Kosch M, Levers A, Lang D, Bartels V, Rahn KH, Pavenstadt H, Hausberg M. A randomized, double-blind study of valsartan versus metoprolol on arterial distensibility and endothelial function in essential hypertension. Nephrol Dial Transplant 2008; 23 . 2280-2285.

30 Mahmud A, Feely J. Reduction in arterial stiffness with angiotensin II antagonist is comparable with and additive to ACE inhibition. Am J Hypertens 2002; 15: 321-325.

31 Morimoto S, Yano Y, Maki K, Sawada K. Renal and vascular protective effects of telmisartan in patients with essential hypertension. Hypertens Res 2006; 29: 567-572.

32 Munakata M, Nagasaki A, Nunokawa T, Sakuma T, Kato H, Yoshinaga K, Toyota T. Effects of valsartan and nifedipine coat-core on systemic arterial stiffness in hypertensive patients. Am J Hypertens 2004; 17: 1050-1055.

33 Nakayama T, Masubuchi Y, Kawauchi K, Masaki R, Hironaga T, Ishima H, Torigoe M, Shimabukuro $\mathrm{H}$. Beneficial effect of beraprost sodium plus telmisartan in the prevention of arterial stiffness development in elderly patients with hypertension and cerebral infarction. Prostaglandins Leukot Essent Fatty Acids 2007; 76: 309-314

34 Rajagopalan S, Kariisa M, Dellegrottaglie S, Bard RL, Kehrer C, Matlow S, Daley W, Pitt B, Brook R. Angiotensin receptor blockade improves vascular compliance in healthy normotensive elderly individuals: results from a randomized double-blind placebocontrolled trial. J Clin Hypertens (Greenwich) 2006; 8: 783-790.

35 Rehman A, Ismail SB, Naing L, Roshan TM, Rahman AR. Reduction in arterial stiffness with angiotensin II antagonism and converting enzyme inhibition. A comparative study among malay hypertensive subjects with a known genetic profile. Am J Hypertens 2007; 20: $184-189$.

36 Suzuki H, Nakamoto $H$, Okada H, Sugahara S, Kanno Y. A selective angiotensin receptor antagonist, Valsartan, produced regression of left ventricular hypertrophy associated with a reduction of arterial stiffness. Adv Perit Dial 2003; 19: 59-66.

37 Takami T, Shigemasa M. Efficacy of various antihypertensive agents as evaluated by indices of vascular stiffness in elderly hypertensive patients. Hypertens Res 2003; 26: 609-614.

38 Feng W. Effect of telmisartan and amlodipine on artery elastic function in essential hypertensive patients. Chin J Clin Res 2011; 1: 810-811.

$39 \mathrm{He} \mathrm{L}$, Wei C, Zheng Z. Impact of ARB on arterial stiffness in female hypertensive patients with impaired glucose tolerance. Chin J Clin Cardiol 2011; 27: 158-159.

40 Huang R, Liu T, Gao Y, Zhen Y. Effect of eplerenone and losartan on arterial stiffness and insulin-like growth factor 1 in patients with mild to moderate essential hypertension. Chin J Clin Cardiol 2013; 29: 64-67.

41 Kong H, Li Y, Zhuang Q, Li X. Effect of benazepril, felodipine and rbesartan on blood pressure and brachial-ankle pulse wave velocity in essential hypertensive patients. Chin Practical Journal of Cardiac Cerebral Pneumal and Vascular Disease 2012; 20. 1657-1658.

$42 \mathrm{Li} \mathrm{Y,} \mathrm{Ma} \mathrm{S.} \mathrm{Comparative} \mathrm{Study} \mathrm{of} \mathrm{telmisartan} \mathrm{and} \mathrm{amlodipine} \mathrm{improving} \mathrm{arterial} \mathrm{stifness}$ in type 2 diabetes mellitus patients with hypertension. Chin J Arterioscler 2013; 21: 252-256

$43 \mathrm{Li} \mathrm{Y,} \mathrm{Shu} \mathrm{M,} \mathrm{Du} \mathrm{M.} \mathrm{Pedndopril,} \mathrm{amlodipine} \mathrm{and} \mathrm{telmisartan} \mathrm{improve} \mathrm{arterial} \mathrm{stifness} \mathrm{in}$ patients with hypertension. Chin J Cardiol 2009; 37: 908-912.

$44 \mathrm{Li} \mathrm{Y}$, Wang $\mathrm{H}$. Effect of various antihypertensive drugs on the improvement of pulse wave velocity in patients with light-to- moderate essential hypertension. Chin New Med 2010; 41: 448-451.

45 Long D, Liu Q. Impact of valsartan on arterial stiffness in patients with coronary heart disease and hypertension. J Clin Res 2010; 27: 436-438.

46 Ruan C, Chen R, Li Z. The change of peripheral vascular function in patients with congestive heart failure and the effect of irbesartan intervention. Med J Chin People Health 2009; 21: 226-228.

47 Ruan C, Chen R, Wang Z. The change of peripheral vascular function in patients with hypertension and the effect of candesartan intervention. Med J Chin People Health 2010; 22: 1069-1070.

48 Wu Z, Qi H, Sheng G. Study on difference of various antihypertensive drugs on pulse wave velocity and correlation with high Sensitivity C-Reactive Protein and von Willebrand Factor. Chin J Arterioscler 2011; 19: 693-696.

49 Yang $\mathrm{P}, \mathrm{Yu} \mathrm{H}$, Wei T. Effects of valsartan on large artery elasticity in patients with essential hypertension. Chin J Exp Tradition Med Formula 2012; 18: 256-259.

50 Zhao W. Effect of valsartan on artery elastic function in elder essential hypertensive patients. Practical Journal of Cardiac Cerebral Pneumal and Vascular Disease 2007; 15: 767-768.

51 Zheng X, Lin J. Effects of various antihypertensive drugs on arterial elasticity in patients with essential hypertension. Chin J Hypertens 2008; 16: 404-407.

52 Weir MR, Dzau VJ. The renin-angiotensin-aldosterone system: a specific target for hypertension management. Am J Hypertens 1999; 12: 205S-213S.

53 Shahin Y, Khan JA, Chetter I. Angiotensin converting enzyme inhibitors effect on arterial stiffness and wave reflections: a meta-analysis and meta-regression of randomised controlled trials. Atherosclerosis 2012; 221: 18-33.

54 Hannah RR, Alexander JS, Michael B. Publication Bias in Meta-Analysis: Prevention Assessment and Adjustments. Wiley, New York, USA, 2005, P105. 